Portland State University

PDXScholar

Mechanical and Materials Engineering Faculty

Publications and Presentations

$10-2016$

\title{
Inverse Structure Functions in the Canonical Wind Turbine Array Boundary Layer
}

\author{
Bianca Viggiano \\ Portland State University \\ Moira Gion \\ Portland State University, mgion@pdx.edu \\ Naseem Ali \\ Portland State University \\ Murat Tutkun \\ Institute for Energy Technology \\ Raúl Bayoán Cal \\ Portland State University, rcal@pdx.edu
}

Follow this and additional works at: https://pdxscholar.library.pdx.edu/mengin_fac

Part of the Materials Science and Engineering Commons, and the Mechanical Engineering Commons Let us know how access to this document benefits you.

\section{Citation Details}

Viggiano, B., Gion, M. S., Ali, N., Tutkun, M., \& Cal, R. B. (2016). Inverse structure functions in the canonical wind turbine array boundary layer. Journal Of Renewable \& Sustainable Energy, 8(5), 1-10. doi:10.1063/ 1.4966228

This Article is brought to you for free and open access. It has been accepted for inclusion in Mechanical and Materials Engineering Faculty Publications and Presentations by an authorized administrator of PDXScholar. Please contact us if we can make this document more accessible: pdxscholar@pdx.edu. 


\title{
Inverse structure functions in the canonical wind turbine array boundary layer
}

\author{
Bianca Viggiano, ${ }^{1}$ Moira S. Gion, ${ }^{1}$ Naseem Ali, ${ }^{1}$ Murat Tutkun, ${ }^{2,3}$ \\ and Raúl Bayoán Cal ${ }^{1}$ \\ ${ }^{1}$ Department of Mechanical and Materials Engineering, Portland State University, \\ Portland, Oregon 97201, USA \\ ${ }^{2}$ Department of Process and Fluid Flow Technology, IFE, Kjeller, Norway \\ ${ }^{3}$ Department of Mathematics, University of Oslo, Blindern, 0316 Oslo, Norway
}

(Received 12 May 2016; accepted 14 October 2016; published online 28 October 2016)

\begin{abstract}
Wind tunnel measurements for a $3 \times 3$ canonical wind turbine array boundary layer are obtained using hot-wire anemometer velocity signals. Two downstream locations are considered, referring to the near- and far-wake, and 21 vertical points are acquired per profile. Velocity increments and exit distances are used to quantify inverse structure functions at both downstream locations. Inverse structure functions in the near-wake show a similar profile for the main vertical locations, but diverge as the moment is increased. In the far-wake, inverse structure functions converge toward a single function for all vertical location and moments. The scaling exponents for inverse structure functions are calculated directly and relatively, using extended self similarity. Scaling exponents show strong dependence on vertical position along the wind turbine profile in the near-wake and remain relatively constant in the farwake. Intermittency in the near-wake is indicated by the nonlinear behavior of the direct and relative scaling exponents when plotted against their respective moments. Published by AIP Publishing. [http://dx.doi.org/10.1063/1.4966228]
\end{abstract}

\section{INTRODUCTION}

Renewable energy will play an increasingly important role as population continues to grow, with the U.S. department of energy projecting that by 2030 the United States will be able to generate $20 \%$ of its electricity from wind energy. ${ }^{1}$ For wind farm power production, the wake of a wind turbine influences the incoming flow for wind turbines behind or adjacent to it. The characterization of the flow in the wake of the wind turbine aids in power production of wind farms. ${ }^{2}$ Lignarolo et al. ${ }^{3}$ studied the near and transitional wake of a model turbine using particle image velocimetry (PIV) and investigated wake flow structures taken directly behind the hub of the turbine. Zhang et $a l .{ }^{4}$ conducted wind tunnel measurements of the wake of a model wind turbine using PIV and hot-wire anemometry velocity measurements. The presence of localized regions of strong vorticity, spatial distributions of turbulence and Reynolds shear stresses as well as changes in velocity due to blade rotation were observed.

With wind farms increasing in size, wakes behind turbine arrays have been further characterized as these interact with the atmospheric boundary layer. Wu and Porté-Agel ${ }^{5}$ studied the interaction between the atmospheric boundary layer and wind farms, comparing aligned and staggered configurations using large-eddy simulation (LES) and verified their results via wind tunnel experiments. Aligned wind farm configurations resulted in higher turbulence intensities behind each turbine at top tip level and less wake interaction with adjacent rows compared to those observed in staggered configurations. Stevens et al. ${ }^{6}$ used field measurements to validate the coupled wake boundary layer (CWBL) model. The CWBL model is in agreement with field measurements, and furthermore, turbulence intensity changes due to thermal effects on wind farm performance. The first and second order moments were used. ${ }^{5,6}$

For further understanding of the turbulent flow in the wake of the wind turbine, observations of higher order moments and characteristics of the signal were carried out by Ali et al., ${ }^{7}$ 
using extended self similarity (ESS) to obtain scaling exponents of the different orders of the structure functions. Exponents varied in the near-wake as a result of the rotor passage, while in the far-wake, scaling exponents remained fairly constant. Ali et al. ${ }^{7}$ compared results to scaling exponents documented in previous studies,${ }^{8-11}$ and found a tendency of ESS to underpredict the exponents.

Statistical properties of canonical wind turbine array boundary layer flow are highly complex and the relationship between the scaling exponents and the statistical moments is nonlinear. The complexity of structure function exponents led Jensen ${ }^{12}$ to present an alternative approach of describing and analyzing a turbulent velocity field. He proposed inverting the ordinary structure function equation and averaging the moments of the distances as a function of a fixed velocity increment. In this framework, a multiscaling spectrum was algebraically formulated with a generated shell model. Because inverse structure functions cover the inertial range to the dissipative range, Biferale et al. ${ }^{13}$ investigated and found a lack of scaling in the inverse structure functions for a dataset measured in a wind tunnel with a Taylor microscale-based Reynolds number, $R e_{\lambda}$, of 2000. In Pearson and van de Water, ${ }^{14}$ scaling with respect to Reynolds number was expected, but algebraic behavior was not. Biferale et al. observed scaling with respect to Reynolds number of a signal filtered by combining successive multiplications of Langevin dynamics to obtain a synthetic multi-affine field. ${ }^{13}$ With experimental data for turbulent wind tunnel flows with $R e_{\lambda}=400-1100$, Pearson and van de Water ${ }^{14}$ established scaling exponents of (relative) inverse structure functions agreeing with the shell model performed by Jensen. ${ }^{12}$ An exact relation between the ordinary and inverse scaling exponents was proposed by Jensen and Roux: ${ }^{15}-\theta(-\zeta(p))=p$, where $\theta$ is the inverse scaling exponent, $\zeta$ is the ordinary scaling exponent, and $p$ is the moment. The relationship holds for a turbulent signal produced by the Gledzer-Ohkitani-Yamada (GOY) shell model $;{ }^{15}$ however, it failed to hold with experimental data from Ref. 14. Zhou et al. ${ }^{16}$ found that the scaling exponents obtained from ESS, $\zeta(p, 2)$, are equal to $p / 2$, for inverse structure functions. This relationship holds for moments less than 3.5.

In this study, the flow behind a wind turbine array is analyzed using inverse structure functions to find the direct and relative scaling exponents at varying wall-normal and streamwise locations. The framework and theoretical analysis are presented in Sec. II and the experimental setup is described in Sec. III. The results and conclusions are discussed in Sec. IV, with a summary of the results in Sec. V.

\section{THEORY}

Kolmogorov suggested the structure of the turbulence cascade describes the transport of turbulence kinetic energy between different scales of motion in a turbulent flow. ${ }^{8}$ The Taylor microscale is defined as $\lambda=\left\langle u_{i}(t)^{2}\right\rangle /\left\langle\left(\partial u_{i}(t) / \partial t\right)^{2}\right\rangle$, where $u_{i}$ is instantaneous velocity, and $t$ is time. In this study, the streamwise component is analyzed; therefore, $u_{i}=u$ and subscript $i$ is dropped in the following equations. Note that Taylor's frozen field hypothesis is implemented in order to relate spatial separation in streamwise direction and time difference. ${ }^{17}$

Structure function of the velocity field examines flow in a turbulent state by considering the velocity difference between two points, raising their difference to the moment, $p$, and studying the variation in space between the points. The kernel of the structure function, the velocity difference, $\Delta u(x, r)$, is computed by subtracting the velocity observed at locations $x+r$ and $x$, meaning $\Delta u(x, r)=u(x+r)-u(x)$. The variation between the measured velocity differences over the spatial separation, $r$, is described by the scaling exponent, $\delta_{p}$, given by

$$
\left\langle[\Delta u(r)]^{p}\right\rangle \sim|r|^{\delta_{p}}
$$

Kolmogorov $^{8}$ suggested that the structure function for the velocity increments at high Reynolds number turbulence has the following form:

$$
\left\langle[\Delta u(r)]^{p}\right\rangle \sim(r)^{p / 3} .
$$


As mentioned in Section I, inverse structure function proposes an alternative approach to study the relation between the physical separation and velocity increment by inverting the ordinary structure function equation and averaging the moments of the distances as a function of the velocity increment. The inverse structure function is defined as

$$
\left\langle[r|\Delta u|]^{p}\right\rangle \sim|\Delta u|^{\xi_{p}}
$$

where $r|\Delta u|$ is the minimal distance from $x$ (the origin in space) that the velocity difference exceeds the velocity increment, $\Delta u$, and $\xi_{p}$ is the scaling exponent for the inverse structure function of moment $p .{ }^{12,27}$ It is suggested, from Kolmogorov, ${ }^{8}$ that the inverted scaling exponent takes the form $\xi_{p}=3 p$. From hereon, $(\cdots)$ is used instead of $|\cdots|$.

Moments are computed using conditional probability density functions (PDF), $P_{\Delta u}(r)$, and are defined by Pearson and van de Water ${ }^{14}$ as

$$
\left\langle[r(\Delta u)]^{p}\right\rangle=\frac{\int_{0}^{\infty} r^{p} P_{\Delta u}(r) d r}{\int_{0}^{\infty} P_{\Delta u}(r) d r},
$$

using histograms of the length scales, $r$.

Scaling exponents for inverse structure functions are also calculated using ESS, introduced by Benzi et al., ${ }^{18}$ by taking the inverse structure function of one moment, $p$, with respect to the inverse structure function of a base moment, $p^{\prime}$, given by

$$
\left\langle[r(\Delta u)]^{p}\right\rangle \sim\left\langle[r(\Delta u)]^{p^{\prime}}\right\rangle^{\xi\left(p, p^{\prime}\right)} .
$$

In this study, $p^{\prime}=2$ was chosen to compare with the findings by Zhou et al. ${ }^{16}$ who used $p^{\prime}=2$ for inverse structure functions. A base moment of $p^{\prime}=3$ was also chosen to compare with the inverted scaling exponent from Kolmogorov, $\xi_{p}=3 p$.

ESS scaling exponents are used to check for intermittency effects in inverse structure functions. Equation (5) can be rearranged as

$$
\xi_{p}=\frac{\log \left([r(\Delta u)]^{p}\right)}{\log \left([r(\Delta u)]^{p^{\prime}}\right)} .
$$

\section{EXPERIMENTAL}

The experiments were carried out in the Corrsin Wind Tunnel at the Johns Hopkins University. The test section of the tunnel is $0.9 \mathrm{~m}$ high, $1.2 \mathrm{~m}$ wide, and $10 \mathrm{~m}$ long. The inflow was conditioned using an active grid, strakes, and wall roughness in the form of sandpaper to create atmospheric-like conditions. The overall layout of the experiment is shown in Figure 1.

The active grid was implemented to generate high levels of free-stream turbulence. The design consists of seven vertical and five horizontal rotating aluminum tubes that contain square $10 \times 10 \mathrm{~cm}^{2}$ winglets of $3.18 \mathrm{~mm}$ thick aluminum plates with circular perforations. The $19 \mathrm{~mm}$ square tubing aluminum shafts were independently actuated and controlled, and were each set to vary direction and rotational speed randomly, changing every second within the range of 210 and $420 \mathrm{rpm}$. Further details about the control of the shafts and actuation process can be found by Kang et al. ${ }^{19}$ Nine strakes, laser cut from acrylic plate, span the inlet of the wind tunnel aimed to obtain a sheared mean velocity profile. The strakes were equally spaced from the sidewalls and $0.1 \mathrm{~m}$ from the leading edge of a $6.7 \mathrm{~m}$ long flat plate. The top of the plate had a layer of 24-grit aluminum oxide sandpaper to contribute to the mean shear profile within the wind tunnel.

A $3 \times 3$ array of wind turbines was placed $3 \mathrm{~m}$ from the leading edge of the rough plate as seen in Figure 1. The distance between successive wind turbines was three diameters in 


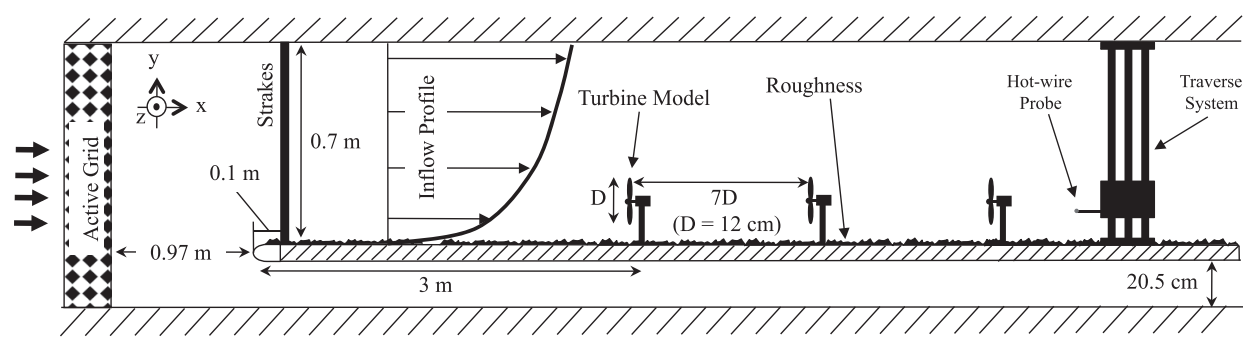

FIG. 1. Experimental setup for the wind turbine array boundary layer. Reproduced with permission from J. Renewable Sustainable Energy 6(2), 023121 (2014). Copyright 2014 AIP Publishing. ${ }^{20}$

crosswind (or spanwise) direction and seven diameters in downwind (or streamwise) direction. The rotating model wind turbines had a $12 \mathrm{~cm}$ rotor diameter and the blades had a $15^{\circ}$ and $10^{\circ}$ twist at the root and the tip, respectively. The ratio of the length scales, corresponding to a reallife rotor diameter and hub height of $100 \mathrm{~m}$, is 830 . The model turbine blade angles were chosen according to a desired angular velocity that corresponded to a field-scale turbine tip speed ratio. Due to the complicated flow field of the experiment, the rotational speeds of the wind turbine models were not uniform across all 9 turbines, differing by $~ 5 \%$. The utilization of rotating blades in the experiment exhibits more realistic characteristics of turbulence intensity and intermittency in the near-wake when compared with experiments performed using stationary disks. ${ }^{21}$ As generators, AC motors of $1 / 4 \mathrm{hp}$ were used to support the rotors at the hub height of $12 \mathrm{~cm}$, cf. Cal $e t a .^{2}$

A cross hot-wire probe was used to obtain vertical velocity profiles behind the last turbine along the centerline at a distance of one rotor diameter, representing the near-wake, and five rotor diameters, representing the far-wake, as seen in Figure 2. Each profile consists of 21 vertical locations spaced by increments of $1 \mathrm{~cm}$ beginning at $0.5 \mathrm{~cm}$ above the floor. The data were recorded at a sampling frequency of $40 \mathrm{kHz}$ for $100 \mathrm{~s}$ at each location.

The mean velocity, Reynolds stress, and power spectra were measured prior to the array to characterize the inflow conditions. Earlier studies using the same dataset showed that there is significant agreement between the streamwise spectra of the inflow and the Kolmogorov power law. ${ }^{2,7,20,22}$ The mean velocity of the incoming flow was $9.4 \mathrm{~m} / \mathrm{s}$. This corresponded to a Reynolds number based on the Taylor microscale, $R e_{\lambda}=u_{r m s} \lambda / \nu$, in the range of 250-1500 depending on the location of measurement. The Taylor microscale and rms velocities change from the point to across the wind turbine array as seen in Figure 3. In the near-wake, $R e_{\lambda}$ fluctuates in the wall-normal direction showing dependence on the wake of the turbine array. The far-wake shows a steady trend of continual increase as the wall-normal position increases, ranging from roughly 500 to 1500 .

Based on the local mean velocity at hub height and the rotor diameter, the Reynolds number for the wind tunnel experiments is $4.5 \times 10^{4}$. This value is on the same order of magnitude of the range where flow statistics in the wakes of wind turbines become independent of the Reynolds number documented by Chamorro et $_{\text {al. }}{ }^{23}$

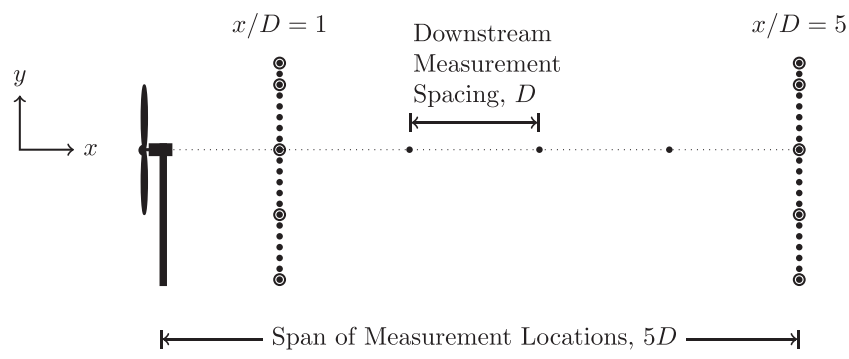

FIG. 2. Measurements taken for the near-wake, $x / D=1$, and far-wake, $x / D=5$, at 21 vertical locations with $\odot$ pertaining to nearest wall, bottom tip, hub height, top tip, and above canopy. 


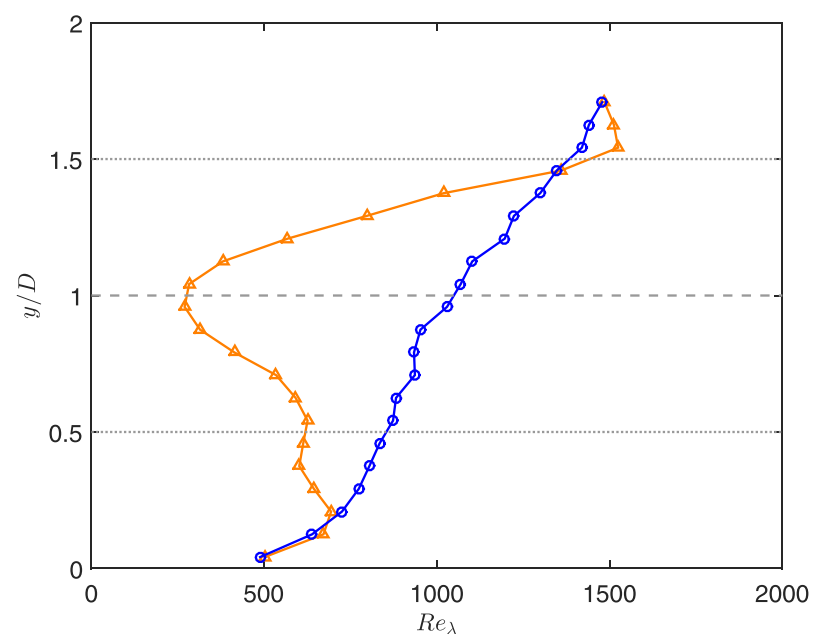

FIG. 3. Streamwise Reynolds number based on the Taylor microscale for near-wake $(\triangle)$ and far-wake $(\bigcirc)$. Dashed lines indicate the highest and lowest blade tip rotation positions $(.$.$) and the hub height location (--)$.

\section{RESULTS}

In Figure 4, inverse structure functions of orders two, four, and six, $p=2,4$, and 6, are displayed as a function of velocity increments for the two downstream positions coinciding with the near- and far-wake at $x / D=1$ and $x / D=5$, respectively. Five vertical positions are selected for each downstream location. More specifically, these locations correspond to the position closest to the wall at $y / D=0.04$, bottom tip $(y / D=0.5)$, hub height $(y / D=1)$, top tip $(y / D=1.5)$, and furthest measured wall-normal position at $y / D=1.7$. An averaged profile of the far-wake curves is taken and used in Figures 4(a), 4(c), and 4(e) for comparison with the near-wake data. The inverse structure functions are normalized by the local Taylor microscale, $\lambda$, and the Taylor microscale-based Reynolds number, $R e_{\lambda}$. Furthermore, the velocity increment, $\Delta u$, is normalized by $\sigma=\sqrt{2\left\langle u^{2}\right\rangle}$, the square root of the large-scale limit of the second-order velocity structure function. ${ }^{24}$ Inverse structure functions of the first, third, and fifth orders are not shown as they follow the trends of the second, fourth, and six orders. Of note, the sixth moment is the highest order that can be computed with good statistical convergence, which was earlier validated by Melius et $a .^{25}$

Inverse structure functions, $\left\langle r(\Delta u)^{p}\right\rangle$, increase monotonically for all vertical locations in both downstream locations with the exception of the hub height in the near-wake. In Figure 4(a), the near-wake region exhibits a similar trend in the five vertical locations with the exception of the hub height, showing slight variation in the curve around $\Delta u / \sigma=0.2$, as well as the location above the canopy, which increases more rapidly than other locations.

Figures 4(c) and 4(e) show similar trends to that of the second order, with increasing variance at the hub height location for small velocity increments in the near-wake. As the order increases, $p$ equaling three through six, the near-wake hub height continues to diverge from the other curves. In addition, the profiles diverge as the velocity increment increases for locations in the near-wake.

Figures 4(b), 4(d), and 4(f) show similar trends for all three orders in the far-wake. The relative standard deviation (RSD), also known as the coefficient of variation, validates the collapse of data in the far-wake for all moments. The highest RSD values occur around $\Delta u / \sigma=$ 0.0325 for all three moments shown, with less variation around $\Delta u / \sigma=0.325$ for the second moment and $\Delta u / \sigma=0.1625$ for the fourth and sixth moments. Furthermore, the RSD values increase as the moment increases, with the mean values of RSD equaling 0.37-0.79.

The tower has a greater effect on the near-wake due to its physical proximity. The modulation caused by the tower decreases with increasing distance behind the turbine. The bottom tip profile in the near-wake diverges while keeping a similar shape due to the effects of mixing 

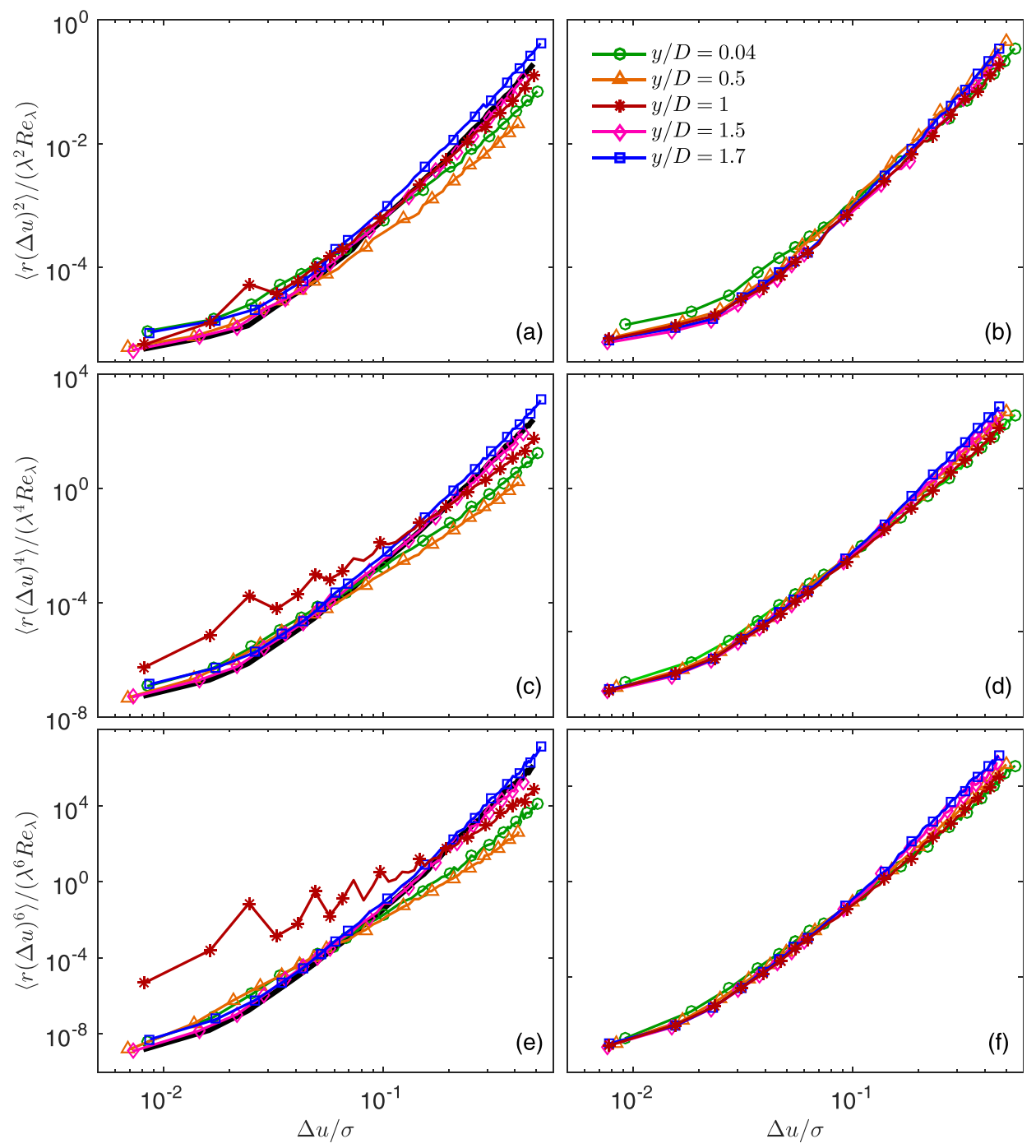

FIG. 4. Inverse structure functions of the second, fourth, and sixth order at wall-normal locations in the near-wake (left column) and far-wake (right-column). An averaged far-wake profile (-) is shown in (a), (c), and (e) for comparison of downstream location.

from the tips of the rotating blades. The divergence of inverse structure functions with respect to the order increase is greatest at the hub height. The deviation observed at hub height for the near-wake is caused by the low Reynolds number and the anisotropy of turbulence at this location being relatively small. ${ }^{22}$ The variation of $R e_{\lambda}$ in the wall-normal direction for the nearwake, shown in Figure 3, indicates the presence of low velocities and smaller length scales from $y / D=0.8$ to $y / D=1.2 .^{20}$ The incoming flow from previous turbines in the array, obstruction of the nacelle, and the mixing of the flow as it passes through the rotating blades are all promoting the decreased velocity and isotropic behavior of the flow. It is also of note that the location of highest anisotropy is the top tip as documented in Ref. 22, and corresponds to the location with strong shearing components and large velocity gradients due to the passage of the blades.

In the near-wake, the collapse of the profiles is less apparent indicating a dependence on factors other than the Taylor microscale. The data varies between the vertical locations. Because the flow above the canopy is above the top tip of the blade, it is relatively undisturbed by the passage of the blades, indicated by the similarity between furthest wall-normal location in the near-wake and the collapsing far-wake data. The collapse of all vertical locations in the 


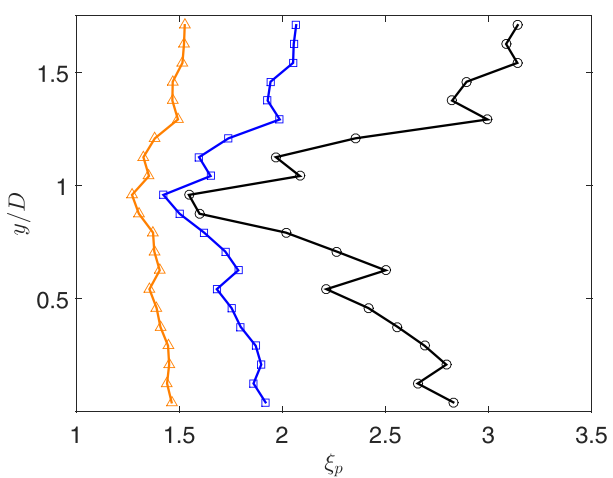

(a) Near-wake for $p^{\prime}=2$

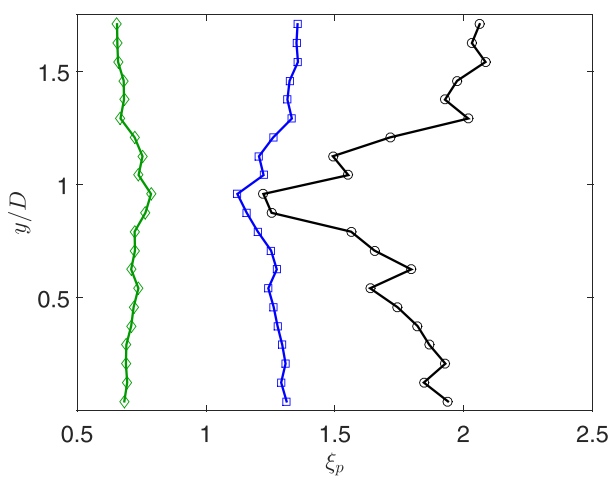

(c) Near-wake for $p^{\prime}=3$

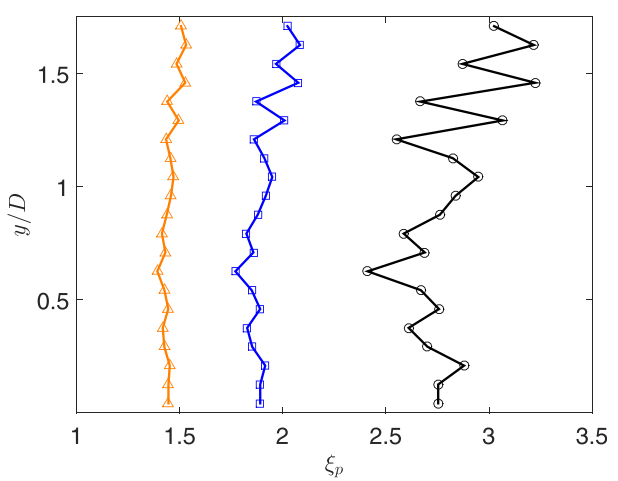

(b) Far-wake for $p^{\prime}=2$

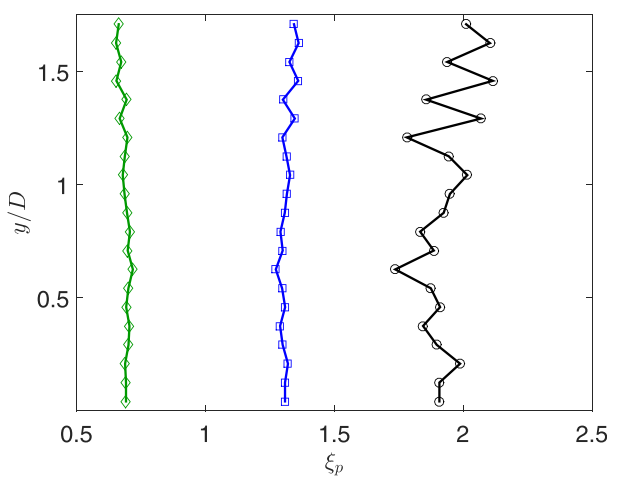

(d) Far-wake for $p^{\prime}=3$

FIG. 5. Scaling exponents for second $(\diamond)$, third $(\triangle)$, fourth $(\square)$, and six $(\bigcirc)$ orders $(p=2, p=3, p=4$, and $p=6)$ plotted against the second and third order base moments $\left(p^{\prime}=2\right.$ and $\left.p^{\prime}=3\right)$.

far-wake indicates the influence of the local Reynolds number and the Taylor microscale on inverse structure functions. In observing the collapse, the ratio of the inverse structure function to these parameters is similar. For the far-wake, all vertical locations show similar behavior as well. More specifically, the far-wake is inhibited from the complexities introduced by the turbine as the flow recovers.

Figures 5(a) and 5(b) show the ESS scaling exponents of the third, fourth, and six order inverse structure functions with respect to the second order, $p^{\prime}=2$, at all vertical locations in the near- and far-wake regions, respectively. Figures 5(c) and 5(d) depict the ESS scaling exponents of the second, fourth, and six order inverse structure functions with respect to the third order, $p^{\prime}=3$. In Figure 5(a), the scaling exponent decreases as the vertical location increases until the hub height location where the scaling exponent is lowest. The scaling exponent then increases as it approaches the above canopy location. In Figure 5(c), the fourth and sixth order curves follow the same trend with the second order showing the opposite tendency. The exponent curves are mirrored about the vertical line of $\xi_{p}=1$. Variations of the curves in the nearand far-wake occur in the same vertical positions, with the magnitude of the variation amplified in the near-wake. For example, the scaling exponent taken at the hub height location differs between the near- and far-wake as well as with the base moment but the general pattern of the variations remains the same. In Figures 5(b) and 5(d), scaling exponents for each order vary within a small range, approximately $5 \%-15 \%$ for $p^{\prime}=2$ and $3 \%-9 \%$ for $p^{\prime}=3$, indicating an agreement between the scaling exponents in the far-wake for $p^{\prime}=2$ and findings by Zhou ${ }^{16}$ of $\zeta(p, 2)=p / 2$ for $p<3.5$. The scaling exponents for the base moment of three, $p^{\prime}=3$, although consistent in the far-wake region, do not agree with the theoretical values of the Kolmogorov theory of $\xi_{p}=3 p$, obtained from inverting the ordinary structure function scaling exponent. In 


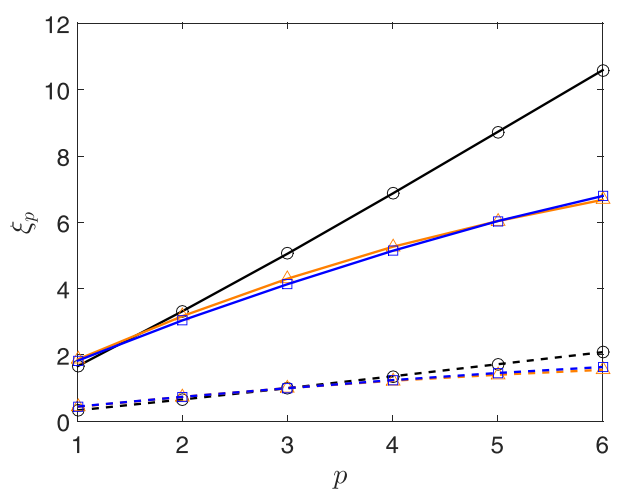

(a) Near-wake

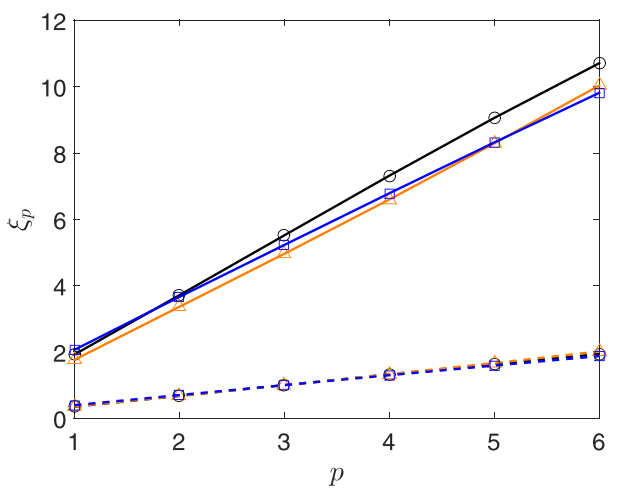

(b) Far-wake

FIG. 6. Direct (-) and relative (--) scaling exponents for the 2nd moment at the bottom tip ( $\square$ ), hub height ( $\triangle$ ), and top tip $(\bigcirc)$ as a function of the moments at (a) near-wake and (b) far-wake.

the near-wake, the effect of the wake is prominent, shown by the dependency of the scaling exponents on their vertical position along the turbine. Furthermore, there is greater variation in the ESS scaling exponents for the near-wake. Far-wake scaling exponents are approximately constant and are not dependent on their vertical location as the variation about the mean is more uniform compared to the near-wake. This agrees with the observations of the inverse structure functions in Figure 4, where the far-wake profiles collapse for higher orders and the near-wake profiles become less compact as the order increases.

In Figures 6(a) and 6(b), the direct and relative scaling exponents are plotted for the nearand far-wake, respectively. For both downstream locations, the direct scaling exponents, calculated from the inverse structure function using Equation (3), are significantly larger and increase more rapidly than the relative scaling exponents, calculated using Equation (6). Ali et al. ${ }^{7}$ observed the same trend, determining that the method of ESS underpredicts the scaling exponents. The direct and relative scaling exponents follow the same profile for each vertical and downstream location, independent of the method. More specifically, the scaling exponents obtained at the bottom tip and hub height are nonlinear in the near-wake for all methods. Figure 6(a) indicates the effect of intermittency at the bottom tip and hub height locations in the near-wake by the nonlinear profile of the scaling exponents. In understanding intermittency found in the near-wake, dynamic loading on turbines can be better identified and consequently the fatigue life and overall performance of a wind farm can be improved. ${ }^{24-26}$ The advanced knowledge of intermittency effects can also aid in placement of turbines within a wind farm, which will also enhance the performance of the wind farm. ${ }^{7}$ In Figure 6(b), a lack of intermittency effects in the far-wake region is validated by the linear profiles of all scaling exponents. The nearest wall and above canopy vertical locations are not shown in Figure 6 for clarity, both profiles in the near- and far-wake are linear. The scaling exponent with respect to the third moment is also not shown in Figure 6, but it follows the same trend as the relative scaling exponent with respect to the second moment.

The scaled conditional probability density functions (PDF), $\alpha \cdot P(r)$, of the length scales are shown for the near- and far-wake in Figures 7(a) and 7(b), respectively, where $\alpha^{2}=\left\langle r^{2}\right\rangle$. Three velocity increments, $\Delta u=0.05 \mathrm{~m} / \mathrm{s}, \Delta u=0.1 \mathrm{~m} / \mathrm{s}$, and $\Delta u=0.5 \mathrm{~m} / \mathrm{s}$, are displayed, which translate to approximately $\Delta u / \sigma=0.0325, \Delta u / \sigma=0.065$, and $\Delta u / \sigma=0.325$, respectively. Furthermore, three vertical locations are shown for each downstream location, namely, bottom tip, hub height, and top tip. Figures 7(c)-7(h) depict $\alpha \cdot P(r) \cdot(r / \alpha)^{p}$ for $p=2,4$, and 6 for the near- and far-wake. Figures $7(\mathrm{c})-7(\mathrm{~h})$ are included to verify the statistical confidence of the orders shown. The convergence of the integrand of $\alpha \cdot P(r) \cdot(r / \alpha)^{p}$, as also investigated by Zhou et al., ${ }^{16}$ indicates that the 6th moment, $p=6$, is not valid for analysis, especially at higher velocity increments. In Figure 7(a), the PDF for the near-wake shows a strong dependence on vertical location for the two lower velocity increments. The top tip, for all velocity increments, 

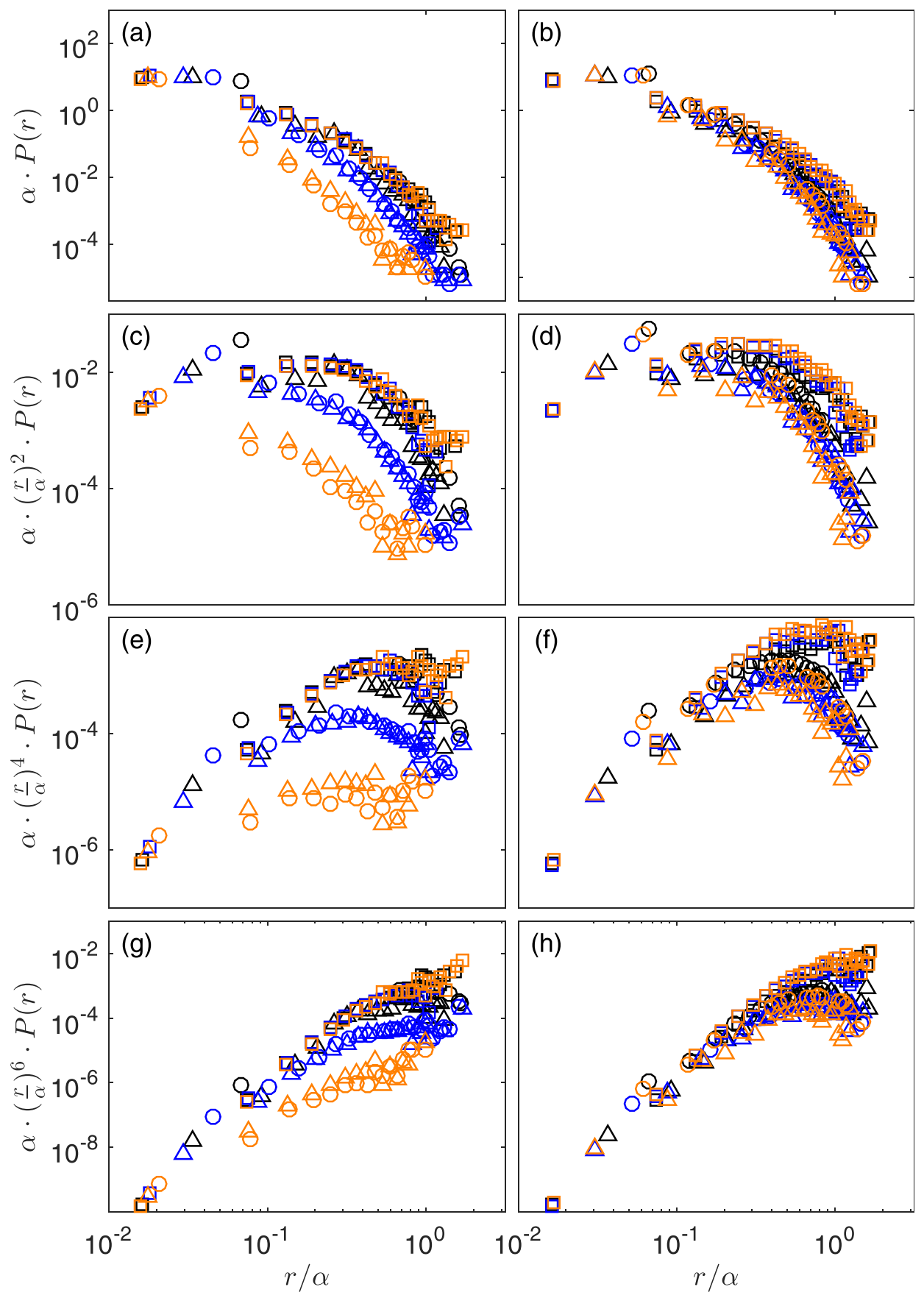

FIG. 7. Scaled probability density function of exit distances, $\alpha \cdot P D F$ and $\alpha \cdot(r / \alpha)^{p} \cdot P D F$ for $p=2,4$, and 6 and for velocity increments $\Delta u=0.05 \mathrm{~m} / \mathrm{s}(\bigcirc), 0.1 \mathrm{~m} / \mathrm{s}(\triangle)$, and $0.5 \mathrm{~m} / \mathrm{s}(\square)$; vertical locations include bottom tip (-), hub height (-), and top tip (-) in near-wake (left column) and far-wake (right column), where $\alpha^{2}=\left\langle r^{2}\right\rangle$.

collapses with the largest velocity increments for all locations. The PDF for the far-wake, Figure 7(b), shows minor dependence on the velocity increment with the $\Delta u=0.5 \mathrm{~m} / \mathrm{s}$ curves diverging slightly from the collapsing profiles for the two lower velocity increments. The succeeding plots of $\alpha \cdot P(r) \cdot(r / \alpha)^{p}$ for the near- and far-wake follow similar trends, with the two lowest velocity increments for the hub height and bottom tip locations diverging for a given vertical location as well as the collapse of all the remaining curves in the near-wake. For the far-wake, the curves of the PDF continue to show a moderate collapse and a small variation for 
the $\Delta u=0.5 \mathrm{~m} / \mathrm{s}$ velocity increment curves, which have a slightly higher probability of the given length scale. It is noted that the PDFs shown have a non-Gaussian distribution due to the velocity increment being only positive.

\section{CONCLUSION}

The relationship between velocity increments and the length scales within a wake initiated by a wind turbine array were studied using inverse structure functions. Inverse structure functions for the near-wake show dependence on parameters introduced by the wind turbine array other than $R e_{\lambda}$. Inverse structure functions in the far-wake collapse for all vertical locations, indicating independence of vertical position along the wind turbine profile. The scaling exponents were found directly from the inverse structure functions. The direct and ESS scaling exponents were compared to one another for the bottom tip, hub height, and top tip with respect to the moment. Scaling exponents in the near-wake for both methods were found to be nonlinear at bottom tip and hub height locations. The scaling exponents in the far-wake were found to be linear, showing a lack of intermittency in the far-wake region. Nonlinear scaling exponents of inverse structure functions indicate that intermittency is present in the near-wake of wind turbine and can be considered for dynamic loading analysis to improve the overall performance of a wind turbine and its fatigue life. Scaled probability density functions of the length scales with respect to $r / \alpha$ in the near-wake diverge in a manner that is dependent on the velocity increment as well as the vertical position while in the far-wake PDF curves for the three vertical positions and velocity increments display moderate collapse. The scaling exponents of inverse structure functions were not inverted quantities of the scaling exponents for ordinary structure functions in this setting. Inverse structure functions are another method to understand the probability of length scales present within the varying velocity increments as larger length scales contain greater amounts of energy.

\section{ACKNOWLEDGMENTS}

This work was in part funded by the National Science Foundation (NSF-CBET-1034581).

\footnotetext{
${ }^{1}$ Wind Vision, "Wind Vision: A New Era for Wind Power in the United States," US Department of Energy, Washington, DC, 2015.

${ }^{2}$ R. B. Cal, J. Lebrón, L. Castillo, H. S. Kang, and C. Meneveau, J. Renewable Sustainable Energy 2(1), 013106 (2010).

${ }^{3}$ L. E. M. Lignarolo, D. Ragni, C. Krishnaswami, Q. Chen, C. J. Simão Ferreira, and G. J. W. Van Bussel, Renewable Energy 70, 31-46 (2014).

${ }^{4}$ W. Zhang, C. D. Markfort, and F. Porté-Agel, Exp. Fluids 52(5), 1219-1235 (2012).

${ }^{5}$ F. Porté-Agel, H. Lu, and Y.-T. Wu, Procedia IUTAM 10, 307-318 (2014).

${ }^{6}$ R. J. A. M. Stevens, D. Gayme, and C. Meneveau, J. Phys.: Conf. Ser. 625(1), 012004 (2015).

${ }^{7}$ N. Ali, A. S. Aseyev, and R. B. Cal, J. Renewable Sustainable Energy 8(1), 013304 (2016).

${ }^{8}$ A. N. Kolmogorov, Proc. Math. Phys. Sci. 32(1), 15-17 (1941).

${ }^{9}$ A. N. Kolmogorov, J. Fluid Mech. 13(01), 82-85 (1962).

${ }^{10}$ U. Frisch, P. L. Sulem, and M. Nelkin, J. Fluid Mech. 87, 719-736 (1978).

${ }^{11}$ Z.-S. She and E. Leveque, Phys. Rev. Lett. 72(3), 336-339 (1994).

${ }^{12}$ M. H. Jensen, Phys. Rev. Lett. 83(1), 76 (1999).

${ }^{13}$ L. Biferale, M. Cencini, D. Vergni, and A. Vulpiani, Phys. Rev. E 60(6), R6295 (1999).

${ }^{14}$ B. R. Pearson and W. van de Water, Phys. Rev. E 71(3), 036303 (2005).

${ }^{15}$ S. Roux and M. H. Jensen, Phys. Rev. E 69, 016309 (2004).

${ }^{16}$ W.-X. Zhou, D. Sornette, and W.-K. Yuan, Phys. D: Nonlinear Phenom. 214(1), 55-62 (2006).

${ }^{17}$ G. I. Taylor, Proc. R. Soc. London A 164(919), 476-490 (1938).

${ }^{18}$ R. Benzi, S. Ciliberto, R. Tripiccione, C. Baudet, F. Massaioli, and S. Succi, Phys. Rev. E 48(1), R29 (1993).

${ }^{19}$ H. S. Kang, S. Chester, and C. Meneveau, J. Fluid Mech. 480, 129-160 (2003).

${ }^{20}$ M. S. Melius, M. Tutkun, and R. B. Cal, J. Renewable Sustainable Energy 6(2), 023121 (2014).

${ }^{21}$ E. H. Camp and R. B. Cal, Phys. Rev. Fluids 1(4), 044404 (2016).

${ }^{22}$ N. Hamilton and R. B. Cal, Phys. Fluids (1994-present) 27(1), 015102 (2015).

${ }^{23}$ L. P. Chamorro, R. E. A. Arndt, and F. Sotiropoulos, Wind Energy 15(5), 733-742 (2012).

${ }^{24}$ M. Tutkun and L. Mydlarski, New J. Phys. 6(1), 49 (2004).

${ }^{25}$ M. S. Melius, M. Tutkun, and R. B. Cal, Phys. D: Nonlinear Phenom. 280, 14-21 (2014).

${ }^{26}$ T. Mücke, D. Kleinhans, and J. Peinke, Wind Energy 14(2), 301-316 (2011).

${ }^{27}$ S. Beaulac and L. Mydlarski, Phys. Fluids 16(6), 2126-2129 (2004).
} 